\title{
Altered expression of microRNAs targeting Dkk-1 in peripheral blood mononuclear cells of patients with ankylosing spondylitis
}

\author{
JINXIAN HUANG ${ }^{1}$, GUOXIANG SONG ${ }^{2}$, ZHIHUA YIN ${ }^{3}$, ZHONGCHAO FU ${ }^{3}$, LIJUN ZHANG ${ }^{l}$ \\ ${ }^{1}$ The University of Hong Kong-Shenzhen Hospital, Guangdong Sheng, China \\ ${ }^{2}$ The Third People's Hospital of Shenzhen, Shenzhen, China \\ ${ }^{3}$ The Fourth People's Hospital of Shenzhen, Shenzhen, China
}

\begin{abstract}
Introduction: Ankylosing spondylitis (AS) is a chronic inflammatory disease characterised by new bone formation, and Dickkopf homologue 1 (Dkk-1) may contribute to the ankylosis of the sacroiliac joint as a main regulator of the Wingless (Wnt) pathway. Increasing evidence shows that microRNAs targeting Dkk-1 might play a critical role in the pathogenesis of rheumatic diseases. We aim to investigate alterations in expression of miRNAs targeting Dkk-1 in AS patients in this study.

Material and methods: The peripheral blood mononuclear cells (PBMCs) of 20 AS patients and 20 normal controls were collected in our study. Three miRNAs targeting DKK1 including miR-29a, miR-335, and $m i R-363$ were selected and quantitative real-time PCR was used to identify the expression of the three miRNAs in these samples. Correlation analysis was conducted between altered miRNA expression and erythrocyte sedimentation rate (ESR), C-reactive protein (CRP), Bath Ankylosing Spondylitis Disease Activity Index (BASDAI), Bath Ankylosing Spondylitis Function Index (BASFI), and mSASSS (modified Stoke Ankylosing Spondylitis Spinal Score).

Results: The expression of miR-29a was significantly higher in AS patients than in healthy controls $(p<0.01)$, while no significance was observed in the expression of miR-335 and miR-363 between $A S$ patients and healthy controls ( $p>0.05)$. No correlation was observed between miR-29a and ESR, CRP, BASDAI, and BASFI $(p>0.05)$. The elevated miR-29a expression was correlated with disease duration and $m S A S S S(p<0.05)$.

Conclusions: MiR-29a might be a useful marker in AS new bone formation and contributes to the regulation of Dkk-1 in Wnt signalling.
\end{abstract}

Key words: ankylosing spondylitis, microRNA, Wnt pathway, Dkk-1.

(Centr Eur J Immunol 2019; 44 (1): 59-64)

\section{Introduction}

Ankylosing spondylitis (AS) is a chronic inflammatory disease characterised by new bone formation that progressively leads to ankylosis and functional disability [1]. Inflammation and bone formation might be two independent processes in AS. Recently developed drugs including biologic agents ameliorated low back pain in most patients, while improvement of clinical symptoms was not necessarily accompanied by retention of radiologic progression. The functional impairment related with bone formation will eventually have a severe effect on quality of life in these patients.

Promising drugs controlling new bone formation are in urgent demand in the treatment of AS.
The Wnt pathway plays a critical role in the new bone turnover of AS. Dickkopf homologue 1 (Dkk-1), an inhibitory molecule which is a main regulator in the Wingless (Wnt) pathway, is dysfunctional in AS patients [2-6].

MicroRNAs (miRNAs) are a class of 18-24-nucleotide noncoding RNAs that play an important role in modulating gene expression. By binding to target mRNA, miRNAs have been found to regulate a variety of physiological functions and contribute to various diseases, including rheumatic diseases. Our study aims to investigate the altered expression of miRNAs targeting DKK1 in the PBMCs of AS patients and identify potential markers contributing to new bone formation in AS.

Correspondence: Jinxian Huang, PhD, The University of Hong Kong-Shenzhen Hospital, $1^{\text {st }}$ Haiyuan Road, 518000, Shenzhen, China, e-mail: huangjx@hku-szh.org

Submitted: 03.04.2017; Accepted: 02.02.2018 


\section{Material and methods}

\section{Study participants}

Twenty AS patients, 20 rheumatoid arthritis (RA) patients, and 20 healthy controls were enrolled in our study. All AS patients in the study, who first visited at Rheumatology Department of our hospitals, fulfilled the modified New York criteria [7]. The enrolled AS patients had spinal involvement and underwent routine treatment of non-steroid anti-inflammatory drugs (NSAIDs) and sulfasalazine. Pelvis, cervical, and lumbar spine X-rays were taken and reviewed independently by two radiologists, for AS diagnosis and mSASSS scoring [8]. RA diagnosis was made according to the 2010 Rheumatoid arthritis classification criteria [9]. All the study protocols and consent forms were approved by the local Ethics Committee. Written permission was obtained from all subjects who participated in the study. Samples from healthy donors were collected from volunteers.

\section{Bioinformatic prediction and database search}

Computational miRNA target prediction analyses were carried out using the algorithms mirTarget2 (http://www. microrna.org/microrna/getGeneForm.do), miRanda (http:// www.microrna.org/microrna/home.do), TargetScan (http:// www.targetscan.org), and PicTar (http://pictar.mdc-berlin. de). "Dkk-1" and "microRNA" were used as keywords in the PubMed search (http://www.pubmed.com) for potential microRNAs targeting Dkk-1.

\section{Quantitative RT-PCR analysis}

Quantitative RT-PCR assays were performed according to the manufacturer's instructions. U6 small nuclear RNA was quantified as a control to normalise differences in total RNA levels. Specific primers of miRNAs selected for real-time PCR were ordered (Applied Biosystems, USA); $\beta$-actin was quantified as the control. Real-time PCR condition was $94^{\circ} \mathrm{C}, 5$-min denaturing; 50 cycles of $94^{\circ} \mathrm{C}$, 15 seconds; $55^{\circ} \mathrm{C}, 30$ seconds. The quantitative RT-PCR was performed by using an Opticon-2 Detection System (Applied Biosystems, Carlsbad, CA, USA). All reactions were run in duplicate and repeated three times. A threshold cycle was determined in the exponential phases of amplification, and the comparative threshold cycle method was calculated with $2^{\Delta \Delta \mathrm{T}}$ and used to calculate the relative gene expression [10]. The value of each control sample was set at one and was used to calculate the fold change in target genes.

\section{Messenger RNA extraction and reverse transcription}

Total RNA was extracted from PBMCs using the Trizol method. The purity of RNA was confirmed by the relative absorbance at 260/280 $\mathrm{nm}$ ratio using a spectrometer.
RNA was reversed to cDNA by Transcriptor First Strand cDNA kit. For real-time quantitative PCR, $10 \mu \mathrm{l}$ $2 \times$ SYBR Green Master mix, $0.4 \mu$ l primer, $1 \mu$ l ROX, $2 \mu \mathrm{l}$ cDNA, and $6 \mu \mathrm{H}_{2} \mathrm{O}$ were mixed to make a $20 \mu \mathrm{l}$ reaction volume. Each sample was run in triplicate. Real-time quantitative PCR was performed at $95^{\circ} \mathrm{C}$ for 10 minutes, followed by 40 cycles at $94^{\circ} \mathrm{C}$ for 15 seconds, $55-60^{\circ} \mathrm{C}$ for 15 seconds, and $72^{\circ} \mathrm{C}$ for 30 seconds.

$\mathrm{Ct}$ value of $\beta$-actin was used as internal control and compared with that of target gene. The $\Delta \Delta \mathrm{Ct}$ method for relative quantity was used as previously described to calculate the differences of expression level for each target gene among samples.

\section{Statistical analysis}

All statistical analyses were carried out in SPSS version 13.0. Data was presented as mean \pm SD. Variables were tested for normality by applying the KolmogorovSmirnov test. Independent-samples $t$ test was used for group comparisons. Spearman correlation was conducted, and a dot plot was drawn when applicable. $p$-values $<0.05$ (two tailed) were considered significant.

\section{Results}

Twenty AS patients and 20 age- and sex-matched healthy controls were included in our study. Characteristics of the study subjects are presented in Table 1. X-ray images showed ankylosis of cervical, lumbar, and spine in the patients (Fig. 1).

The threshold cycle $(\mathrm{Ct})$ value denotes how many PCR cycles are required for the sample fluorescence to reach the threshold level. Thus, the more target microRNA present in a sample, the lower the $\mathrm{Ct}$ value will be, because the threshold is reached sooner. The expression of miR-29a was significantly higher in AS patients than that in RA patients and normal controls $(\Delta \mathrm{Ct}: 9.53 \pm 1.90$ vs. 11.31 \pm 0.95 and $11.71 \pm 1.16, p<0.001$, Fig. $2 \mathrm{~A})$. The difference of miR-335 and miR-363 expression between AS patients, RA patients, and normal controls was not significant $(\Delta \mathrm{Ct}$ : $10.59 \pm 1.79$ vs. $10.14 \pm 1.23$ and $11.06 \pm 0.54, p>0.05$; $\Delta \mathrm{Ct}: 5.30 \pm 2.92$ vs. $6.05 \pm 0.98$ and $5.37 \pm 1.48, p>0.05$, respectively, Fig. 2B-C). The fold change of altered expression of these miRNAs in AS patients and normal controls was also shown in Table 2. The expression of DKK1 between AS patients, RA patients, and normal controls was not significant $(\Delta \mathrm{Ct}: 0.88 \pm 0.66$ vs. $0.95 \pm 0.87$ and 0.99 $\pm 0.64, p>0.05)$.

No correlation was observed between miR-29a and DKK1 $(r=-0.074, p=0.758)$, ESR $(r=-0.331$, $p=0.074), \mathrm{CRP}(r=-0.259, p=0.168), \mathrm{BASIDAI}(r=-0.100$, $p=0.676)$, and BASFI $(r=-0.127, p=0.595)$. The miR-29a $\mathrm{Ct}$ value was correlated with disease duration $(r=-0.579$, $p=0.008$, Fig. 3A) and mSASSS $(r=-0.621, p=0.003$, 
Table 1. Characteristics of the study subjects

\begin{tabular}{|c|c|c|}
\hline Factor & $\begin{array}{c}\text { Ankylosing } \\
\text { spondylitis } \\
\text { patient group, } \\
n=\mathbf{2 0}\end{array}$ & $\begin{array}{l}\text { Healthy } \\
\text { control } \\
\text { group, } \\
n=20\end{array}$ \\
\hline Age (years), mean \pm SD & $32.95 \pm 8.23$ & $31.78 \pm 7.31$ \\
\hline No. (\%) male & $80 \%$ & $80 \%$ \\
\hline HLA-B27 positive no. $(\%)$ & $90 \%$ & NA \\
\hline $\operatorname{ESR}(\mathrm{mm} / \mathrm{h})$, mean $\pm \mathrm{SD}$ & $49.95 \pm 15.12$ & NA \\
\hline $\mathrm{CRP}(\mathrm{mg} / \mathrm{l})$, mean $\pm \mathrm{SD}$ & $22.94 \pm 10.93$ & NA \\
\hline BASDAI & $6.10 \pm 0.84$ & NA \\
\hline BASFI & $66.35 \pm 5.91$ & NA \\
\hline mSASSS & $15.05 \pm 4.58$ & NA \\
\hline $\begin{array}{l}\text { Disease duration (years), } \\
\text { mean } \pm \text { SD }\end{array}$ & $4.85 \pm 3.53$ & NA \\
\hline
\end{tabular}

Fig. 3B). Meanwhile, disease duration was positively correlated with mSASSS $(r=0.473, p=0.035$, Fig. 3C).

\section{Discussion}

AS is an inflammatory disease that predominantly affects axial joints and inter-vertebral spaces. Sacroiliitis is the hallmark of AS. The disease is characterised by tight interplay between chronic inflammation and bone formation, which is only partly understood.

The mechanisms controlling new bone formation in AS are not yet fully defined, but some evidence indicates that the Wnt pathway may be the main regulator of this process. DKK-1, which can be induced by TNF and is a key inhibitor to the classic Wnt pathway, could be a link between inflammation and bone formation processes in AS [11]. The balance between proteins involved in bone formation such as Wnt proteins and that suppressing bone formation such as DKK is apparently one of the decisive steps in joint remodelling, particularly in determining whether an affected joint faces erosive damage or build up of bony spurs [12].

Dkk-1 is an inhibitory molecule that regulates the Wnt pathway, which controls osteoblastogenesis. Dkk-1 suppresses bone formation by interfering with the Wnt pathway. Dkk-1 is essential for the maintenance of skeletal homeostasis as an inhibitor of Wnt signalling and osteogenic differentiation. DKK-1 is a regulator of bone mass, with increased expression linked to osteopaenia and decreased expression to high bone mass.

Evidence that Dkk-1 is dysfunctional in AS was proven by several studies [2-6]. Dkk-1 level is lower in patients with AS than in healthy people, which might be related to the pathogenesis of new bone formation in AS. AS patients

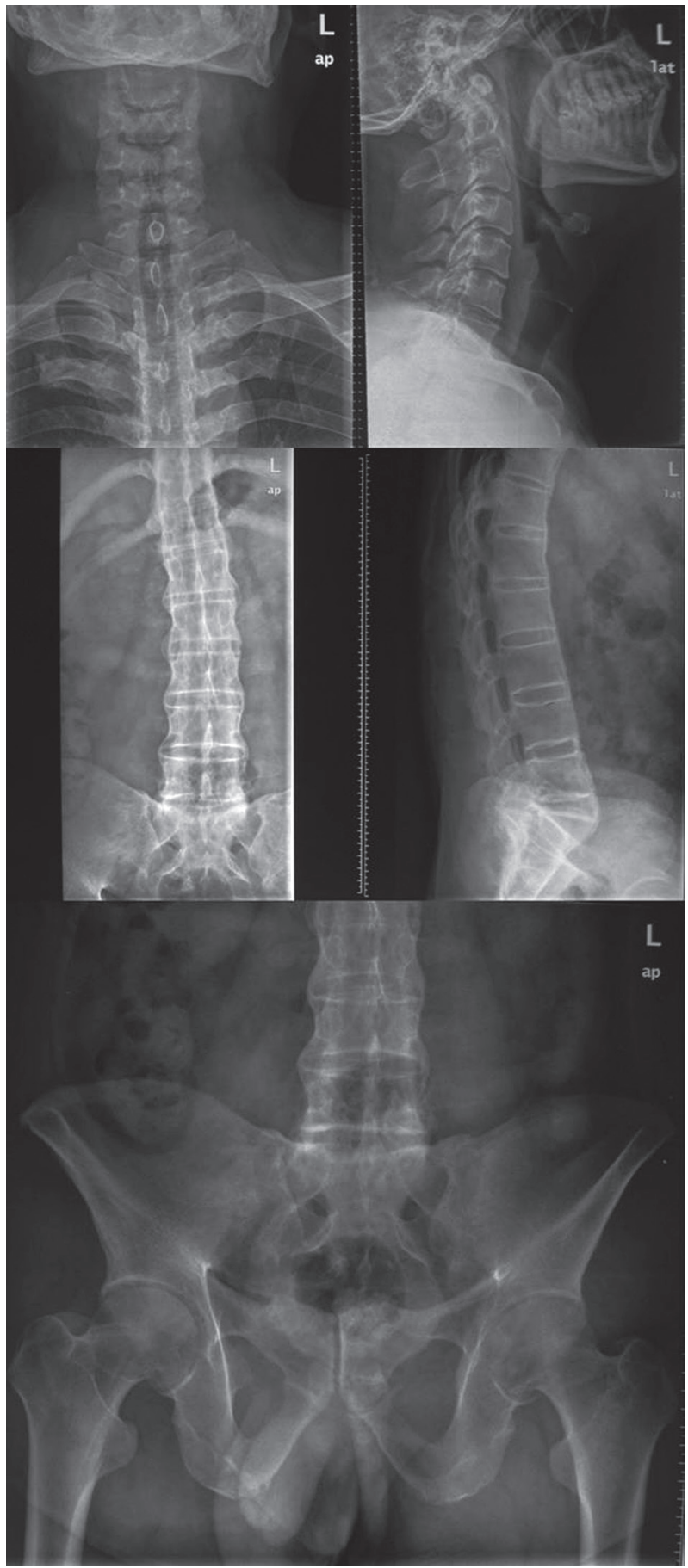

Fig. 1. A patient with advanced ankylosis shown in cervical, lumbar, and pelvis X-ray images. Cervical, lumbar, and pelvis X-ray images taken in a patient for diagnosis and mSASSS (modified Stoke Ankylosing Spondylitis Spinal Score) scoring

with no syndesmophyte formation show significantly higher functional Dkk1 levels, suggesting that blunted Wnt signalling suppresses new bone formation and consequently 

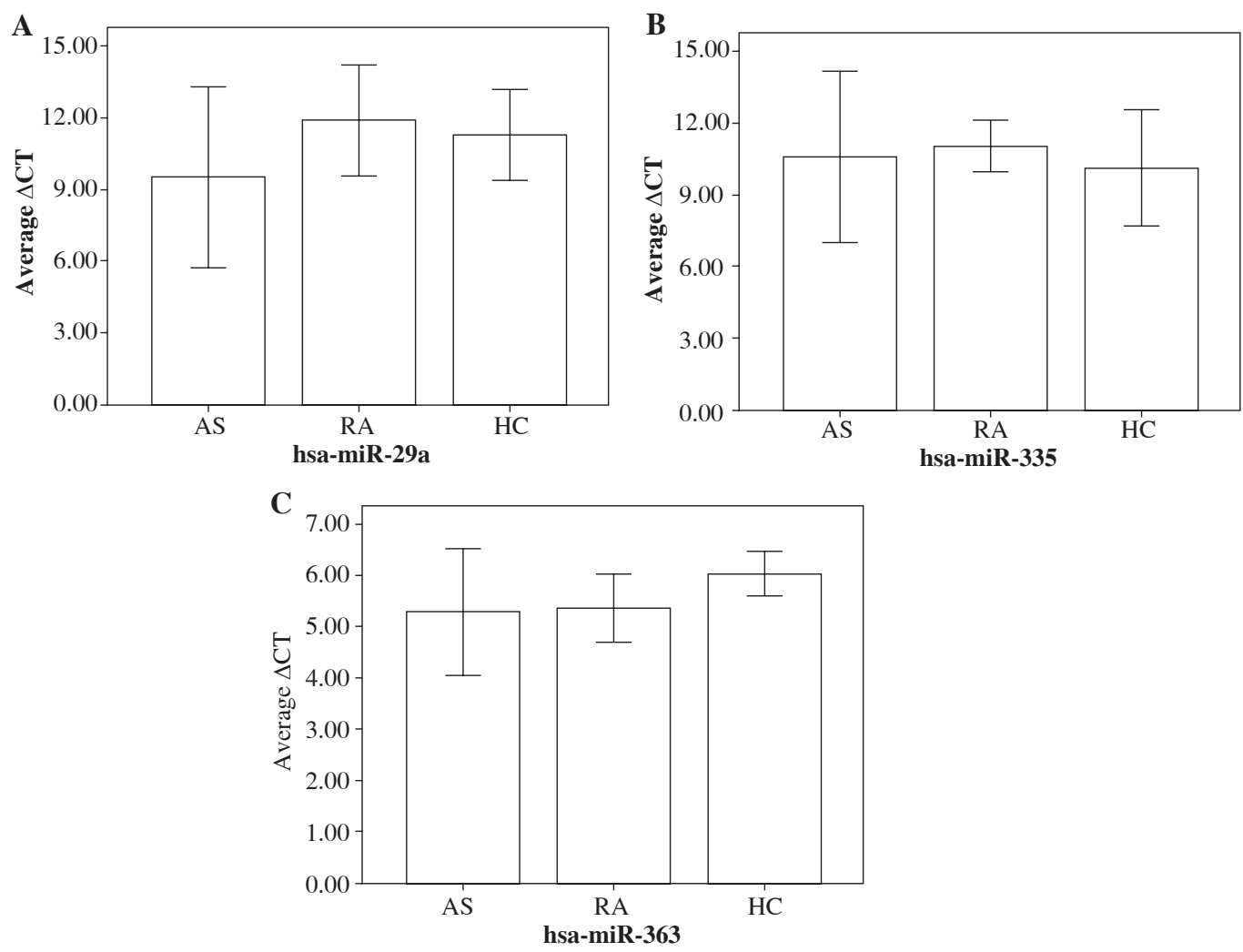

Fig. 2. Average delta Ct value of 3 microRNAs targeting Dkk-1 in ankylosing spondylitis (AS) patients and healthy controls. Results of miR-29a (A), miR-335 (B), and miR-363 (C) expression shown as Ct value (mean \pm SD) in AS patients and healthy controls (HC) measured by qRT-PCR

Table 2. Altered miRNAs in ankylosing spondylitis patients and normal controls

\begin{tabular}{lccc}
\hline Group & hsa-miR-29a & hsa-miR-335 & hsa-miR-363 \\
\hline Ankylosing spondylitis patients, average $\Delta \mathrm{C}_{\mathrm{T}}(n=20)$ & $9.53 \pm 1.90$ & $10.59 \pm 1.79$ & $5.30 \pm 2.92$ \\
\hline Normal controls, average $\Delta \mathrm{C}_{\mathrm{T}}(n=20)$ & $11.71 \pm 1.16$ & $11.06 \pm 0.54$ & $5.37 \pm 1.48$ \\
\hline$\Delta \Delta \mathrm{C}_{\mathrm{T}}$ & $-2.38 \pm 2.23$ & $-0.47 \pm 1.87$ & $-0.07 \pm 3.27$ \\
\hline $2^{-\Delta \Delta_{\mathrm{CT}}}$ & $5.21(1.11 \sim 24.42)$ & $1.39(0.38 \sim 5.06)$ & $1.05(0.11 \sim 10.13)$ \\
\hline$p$ value & 0.00003857 & 0.281 & 0.917 \\
\hline
\end{tabular}

syndesmophyte growth and spinal ankylosis [13]. Similar to serum sclerostin levels, the functional Dkk1 level thus emerges as a potential biomarker for structural progression in patients with AS. Blockade of DKK1 promotes ankylosis of the sacroiliac joint [14]. DKK1 influences inflammatory of sacroiliac joints by prevention of joint ankylosis. This may indicate an important role of the Wnt signalling pathway in the structural bone changes of axial joint disease.

Increasing evidence indicates that aberrant expression of miRNAs is implicated in the pathogenesis of autoimmune diseases. We used the PubMed search tool combined with bioinformatics analysis as a strategy for screening potential miRNAs targeting DKK-1. Three miRNAs in- cluding miR-29a, miR-335, and miR-363 were selected for subsequent study.

Dkk-1 is a direct target of miR-29a. MiR-29 promotes osteoblastic differentiation, and its ability to down-regulate the expression of key Wnt signalling inhibitor Dkk-1 likely contributes to this effect by promoting Wnt signalling [15]. MiR-363 was increased more than two-fold by a change in CD4+ T cells of SF from rheumatoid arthritis (RA) patients, whereas miR-29a was significantly downregulated in a previous microarray data, with a possibly similar bone turnover mechanism involved [16]. MiR-335-5p activates Wnt signalling and promotes osteogenic differentiation by down-regulating DKK1. MiR-335 might interact with Dkk-1 in canonical Wnt signalling pathway, 
A

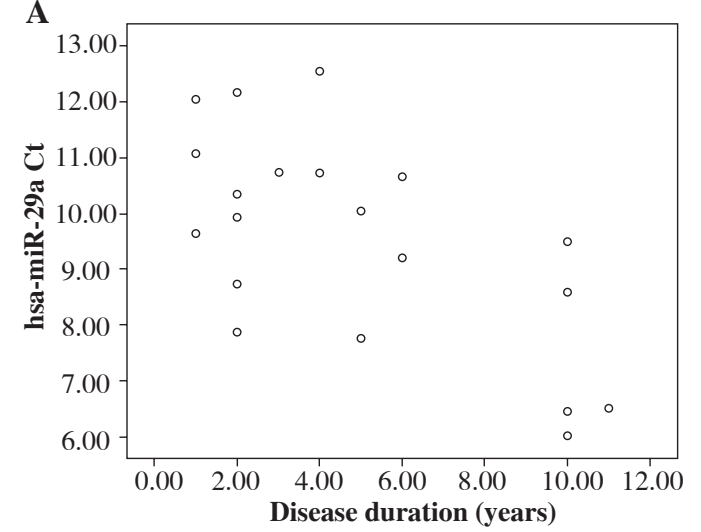

B

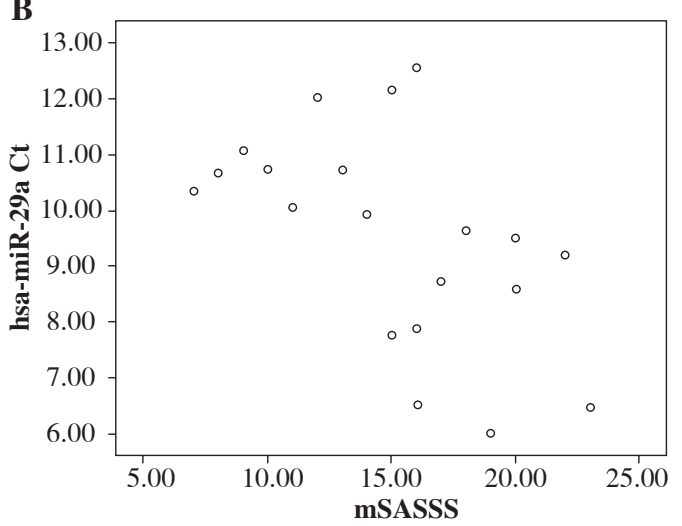

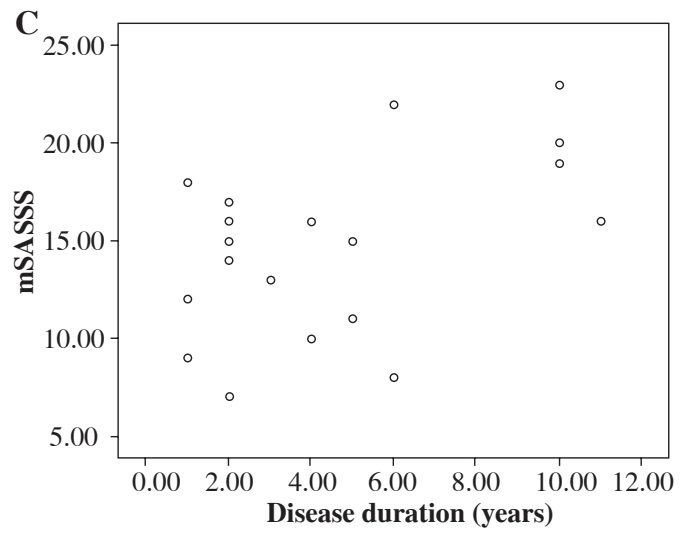

Fig. 3. Correlation between miR-29a, disease duration, and mSASSS (modified Stoke Ankylosing Spondylitis Spinal Score). Positive correlation was observed between miR-29a and disease duration (A), miR-29a and mSASSS (B), as well as disease duration and mSASSS $(\mathbf{C})$

indicating that DKK-1 could be a potential target gene of miR-335 [17, 18].

Our results showed that only miR-29a was significantly and specifically elevated in AS patients, as compared to disease controls (RA patients) and normal controls. And the elevated miR-29a expression is positively correlated with disease duration and mSASSS. Because the mSASSS is an index reflecting structural changes weighting on new bone formation and indicating radiologic progression, the elevated miR-29a expression accompanied by advanced radiologic progression with prolonged disease duration might imply the contribution of miR-29a to new bone formation in AS.

One limitation of our study is the small sample size enrolled. The preliminary results require further investigation. The expression of miR-29a was not negatively correlated with DKK1 as expected, and miR-29a might target dozens of other genes. Meanwhile, further studies should focus on whether miR-29a can suppress the expression of Dkk-1 using western blotting as well as in vitro functional studies, in order to obtain more convincing data for the miR-29a modulation on Dkk-1.
In conclusion, miR-29a might be a specific microRNA for AS, which might contribute to the new bone formation of AS by targeting DKK-1.

\section{Acknowledgments}

This research was supported by National Natural Science Foundation of China (81301529), Natural Science Foundation of Guangdong Province (S2013040012296), Shenzhen science and technology project (JCYJ2015 0331142757389), Science and Technology Planning Project of Guangdong Province (2014A020212617), and Medical Scientific Research Foundation of Guangdong Province (A2015395).

The authors declare no conflict of interest.

\section{References}

1. Van Der Heijde D, Landewé R, Einstein S, et al. (2008): Radiographic progression of ankylosing spondylitis after up to two years of treatment with etanercept. Arthritis Rheum 58: 1324-1331. 
2. Kwon SR, Lim MJ, Suh CH, et al. (2012): Dickkopf-1 level is lower in patients with ankylosing spondylitis than in healthy people and is not influenced by anti-tumor necrosis factor therapy. Rheumatol Int 32: 2523-2527.

3. Daoussis D, Liossis SN, Solomou EE, et al. (2010): Evidence that Dkk-1 is dysfunctional in ankylosing spondylitis. Arthritis Rheum 62: 150-158.

4. Sui L, Zhang K, Wang Y (2011): Clinical Significance of Serum Level of Dkk-1 in Patients with Ankylosing Spondylitis. Tianjin Med J 39: 918-920.

5. Taylan A, Sari I, Akinci B, et al. (2012): Biomarkers and cytokines of bone turnover: extensive evaluation in a cohort of patients with ankylosing spondylitis. BMC Musculoskelet Disord 13: 191.

6. Hu Z, Xu M, Li Q, et al. (2012): Adalimumab significantly reduces inflammation and serum DKK-1 level but increases fatty deposition in lumbar spine in active ankylosing spondylitis. Int J Rheum Dis 15: 358-365.

7. Van Der Linden S, Valkenburg HA, Cats A (1984): Evaluation of diagnostic criteria for ankylosing spondylitis. A proposal for modification of the New York criteria. Arthritis Rheum 27: 361-368.

8. Creemers MC, Franssen MJ, van't Hof MA, et al. (2005): Assessment of outcome in ankylosing spondylitis: an extended radiographic scoring system. Ann Rheum Dis 64: 127-129.

9. Aletaha D, Neogi T, Silman AJ, et al. (2010): 2010 Rheumatoid arthritis classification criteria: an American College of Rheumatology/European League Against Rheumatism collaborative initiative. Arthritis Rheum 62: 2569-2581.

10. Livak KJ, Schmittgen TD (2001): Analysis of relative gene expression data using real-time quantitative PCR and the 2(-Delta Delta C(T)) Method. Methods 25: 402-408.

11. Daoussis D, Andonopoulos AP (2011): The emerging role of Dickkopf-1 in bone biology: is it the main switch controlling bone and joint remodeling? Semin Arthritis Rheum 41: 170-177.

12. Schett G, Landewé R, van der Heijde D (2007): TNF blockers and structural remodeling in ankylosing spondylitis-what is reality and what is fiction? Ann Rheum Dis 66: 709-711.

13. Heiland GR, Appel H, Poddubnyy D, et al (2012): High level of functional dickkopf-1 predicts protection from syndesmophyte formation in patients with ankylosing spondylitis. Ann Rheum Dis 71: 572-574.

14. Uderhardt S, Diarra D, Katzenbeisser J, et al (2010): Blockade of Dickkopf (DKK)-1 induces fusion of sacroiliac joints. Ann Rheum Dis 69: 592-597.

15. Kapinas K, Kessler C, Ricks T, et al (2010): miR-29 modulates Wnt signaling in human osteoblasts through a positive feedback loop. J Biol Chem 285: 25221-25231.

16. Li J, Wan Y, Guo Q, et al (2010): Altered microRNA expression profile with miR-146a upregulation in CD4+ T cells from patients with rheumatoid arthritis. Arthritis Res Ther 12: R81.

17. Zhang J, Tu Q, Bonewald LF, et al (2011): Effects of miR$335-5 p$ in modulating osteogenic differentiation by specifically downregulating Wnt antagonist DKK1. J Bone Miner Res 26: 1953-1963.

18. Tomé M, López-romero P, Albo C, et al (2011): miR-335 orchestrates cell proliferation, migration and differentiation in human mesenchymal stem cells. Cell Death Differ 18: 985-995. 